The ASTROPHYSICAL JOURNAL SUPPLEMENT SERIES, 90:949-953, 1994 February

(C) 1994. The American Astronomical Society. All rights reserved. Printed in U.S.A.

\title{
RELATIVISTIC PARTICLE TRANSPORT IN HOT ACCRETION DISKS
}

\author{
Peter A. Becker and Menas Kafatos \\ Department of Physics and Institute for Computational Sciences and Informatics, George Mason University, Fairfax, VA 22030-4444 \\ AND \\ MiChaEl MAISACK \\ Astronomical Institute, University of Tuebingen, Germany \\ Received 1993 March 4; accepted 1993 July 9
}

\begin{abstract}
Accretion disks around rapidly rotating black holes provide one of the few plausible models for the production of intense radiation in AGNs above energies of several hundred $\mathrm{MeV}$. The rapid rotation of the hole increases the binding energy per nucleon in the last stable orbit relative to the Schwarzschild case, and naturally leads to ion temperatures in the range $10^{12}-10^{13} \mathrm{~K}$ for sub-Eddington accretion rates. The protons in the hot inner region of a steady, two-temperature disk form a reservoir of energy that is sufficient to power the observed EGRET outbursts if the black hole mass is $10^{10} M_{\odot}$. Moreover, the accretion timescale for the inner region is comparable to the observed transient timescale of $\sim 1$ week. Hence EGRET outbursts may be driven by instabilities in hot, two-temperature disks around supermassive black holes. In this paper we discuss turbulent ( stochastic) acceleration in hot disks as a possible source of $\mathrm{GeV}$ particles and radiation. We constrain the model by assuming the turbulence is powered by a collective instability that drains energy from the hot protons. We also provide some ideas concerning new, high-energy Penrose processes that produce $\mathrm{GeV}$ emission be directly tapping the rotational energy of Kerr black holes.
\end{abstract}

Subject headings: acceleration of particles — accretion, accretion disks - galaxies: nuclei quasars: individual (3C 279) — radiation mechanisms: nonthermal

\section{INTRODUCTION}

The physical nature of the central power source in active galactic nuclei (AGNs) and quasars (QSOs) has remained one of the most challenging problems in modern astrophysics. At present, the most favored model for the central engine is the supermassive black hole hypothesis (Lynden-Bell 1969; Rees 1984; Kafatos 1988). In this scenario, gas falling onto a central black hole radiates with a luminosity which for disk accretion can exceed a few percent of $\dot{M} c^{2}$, where $\dot{M}$ is the accretion rate. Despite the attractiveness of the accretion-powered supermassive black-hole hypothesis, a definitive test of this hypothesis remains elusive. Support for it has emerged for only a handful of galactic nuclei including the nuclei of the nearby "inactive" galaxies M31 and M32 (Kormendy 1988, 1991). Yet, numerous observational results have tied AGNs together, implying that the same fundamental power source is at work in all active nuclei. In this way the relative simplicity of the black-hole hypothesis has received indirect support.

The recent detection by EGRET (Hartman et al. 1992) and COMPTEL (Hermsen et al. 1993) on board the Compton Gamma Ray Observatory (CGRO) of high-energy $\gamma$-rays from over two dozen blazars has called into question the viability of a number of theoretical models. In particular, observations of $3 \mathrm{C} 279$ spanning the energy range from $1 \mathrm{MeV}$ to several $\mathrm{GeV}$ with a luminosity of $\sim 10^{48} \mathrm{ergs} \mathrm{s}^{-1}$ impose severe constraints on production mechanisms for high-energy radiation in active galaxies. The observed luminosity implies a central mass close to $10^{10} M_{\odot}$ if the emission is unbeamed and the source is radiating near its Eddington limit. As we shall argue below, hot two-temperature accretion disks may provide the only plausible alternative to relativistic inverse-Compton beam models, which require extremely high bulk Lorentz factors in order to explain the 3C 279 observations. Furthermore, hot disks may prove to be the only viable mechanism for producing EGRET emission in nonbeamed AGNs, should this be discovered.

It is widely accepted that the blazar phenomenon is associated with jets of plasma moving with relativistic velocities closely aligned with our line of sight. If the jets emanate along the rotational axis of the black hole, then it follows that the accretion disks thought to be present in these systems are being viewed nearly face-on. This is true whether the high-energy emission actually arises in the jet or not. Since the physics of jets is not well understood and a number of ad hoc assumptions need to be made, it is important to examine emission from the disk itself as an alternative or possible supplement to relativistic beam models for the production of the observed high-energy radiation. Whether beams or hot disks produce the $\gamma$-rays seen by the $C G R O$, the ultimate energy source must be attributed to accretion. As such, one may envisage a scenario where both beams and disks are required to explain the full spectrum of high-energy AGN emission. This makes an understanding of the high-energy emission properties of disks crucial for the interpretation of $C G R O$ data.

The paper is organized as follows. In $\S 2$ we briefly review the major features of hot accretion disks. In $\S 3$ we discuss the results obtained for the steady state pair distribution and the inverse-Compton spectrum by considering only particle injection, escape, and losses. In $\S 4$ we discuss Penrose processes as a potential source of $\mathrm{GeV}$ particles and radiation. In $\S 5$ we ex- 
plore stochastic acceleration in hot disks as an alternative source of $\mathrm{GeV}$ electrons and positrons. We conclude in $\$ 6$ with a summary and conclusions.

\section{HOT ACCRETION DISKS IN AGNs}

The high radiative efficiency of blackbody cooling leads to temperatures in optically thick disks on the order of $T_{\text {thick }} \sim$ $10^{6}-10^{8} \mathrm{~K}$ that are far too low to explain observations of $\gamma$-ray emission in galactic and extragalactic sources (Shapiro, Lightman, \& Eardley 1976, hereafter SLE). On the other hand, in two-temperature accretion disks, the protons can reach nearvirial temperatures $\left(T_{\text {virial }} \sim 10^{10}-10^{11} \mathrm{~K}\right.$ for Schwarzschild, or $\sim 10^{12}-10^{13} \mathrm{~K}$ for canonical Kerr), while the electron temperature remains two or three orders of magnitude lower due to efficient cooling. The maintenance of the strong temperature contrast between the protons and the electrons requires that the protons cool on timescales long compared with the inflow timescale (SLE). If the protons cool exclusively via Coulomb interactions with the electrons, then two-temperature accretion flows can exist provided the accretion rate $\dot{M} \leqslant$ $50 \alpha^{2} \dot{M}_{\mathrm{E}}$ (Rees et al. 1982), where $\dot{M}_{\mathrm{E}}=L_{\mathrm{E}} / c^{2}\left(L_{\mathrm{E}}\right.$ is the Eddington luminosity) and $\alpha \leq 1$ is the viscosity parameter (e.g., Pringle 1981; Shakura \& Sunyaev 1973). The limit on the accretion rate is lower in the presence of $e^{+} e^{-}$pairs (Begelman, Sikora, \& Rees 1987) or in regions of large-amplitude, small-scale MHD turbulence (Begelman \& Chiueh 1988).

Eilek (1980) and Eilek \& Kafatos (1983, hereafter EK) extended the work of SLE, which was based on the Schwarzschild geometry, to model steady, two-temperature accretion disks around canonical Kerr black holes. The protons in the hot inner region achieve temperatures on the order of the virial temperature, $T_{p} \leqslant T_{\text {virial }} \sim 10^{12}-10^{13} \mathrm{~K}$. At these high temperatures, a significant fraction of the protons have energy $E>290$ $\mathrm{MeV}$ above the threshold for pion production via proton-proton collisions. The decay of pions created in the hot region as a result of proton-proton collisions can produce primary $\gamma$-rays with energies up to $\sim 500 \mathrm{MeV}$, as well as significant quantities of relativistic $e^{+} e^{-}$pairs with Lorentz factors in the range $10<$ $\gamma<300$. In the EK model, roughly half of the bolometric luminosity is produced in the inner region in the form of primary high-energy radiation and/or electron-positron secondary radiation. $C G R O$ observations will prove crucial for discriminating between hot-disk and beam models and determining source parameters, since the power emitted per decade of energy peaks in the $\gamma$-ray region for many of the EGRET-bright AGNs which have been detected. We point out that the observed high degree of alignment is not inconsistent with a disk origin for the $\gamma$-rays, since the $\gamma-\gamma$ pair production optical depth is expected to increase rapidly as a function of the propagation angle measured from the rotation axis. In $\S 5$ we discuss stochastic processes that may be capable of accelerating electrons and positrons to the $\mathrm{GeV}$ energies required to explain the highest-energy EGRET observations.

\section{GAMMA-RAYS FROM RELATIVISTIC PAIRS}

In the hot, two-temperature disk model, high-energy particles and $\gamma$-rays arise as a natural consequence of accretion onto rapidly-spinning black holes. The relativistic $e^{+} e^{-}$pairs injected with Lorentz factors in the range $10<\gamma<300$ will experience a number of different collision, acceleration, and loss processes while in the disk. As a first approach, we have modeled the energy distribution of the relativistic pairs by solving a simple, time-independent transport equation for the Green's function describing the effects of monoenergetic particle injection, particle escape at the speed of light (using an escape probability formalism), and losses due to synchrotron and inverse-Compton emission in a homogeneous and isotropic plasma. The steady state particle distribution $N_{S}(\gamma)$ obtained by integrating the Green's function over the flat-top energy spectrum of the injected pairs approaches the form $N_{S}(\gamma) \propto \gamma^{-2}$ for cases involving strong radiative cooling, as is expected in the nuclei of luminous active galaxies. The resulting inverse-Compton emission appears in the range $\sim 1 \mathrm{keV}-4$ $\mathrm{MeV}$ if the pairs are exposed to UV radiation, or in the range $\sim 40 \mathrm{keV}-150 \mathrm{MeV}$ if the pairs are exposed to X-rays. In Figure 1 , we display plots obtained by fitting the simple model to multifrequency OSSE, COMPTEL, and EGRET data for four AGNs, including 3C 279 (see also Maisack, Becker, \& Kafatos 1994). In general, the hot-disk model predicts an anticorrelation between the OSSE and EGRET signals due to $\gamma-\gamma$ optical depth considerations. The lack of emission above several hundred $\mathrm{MeV}$ reflects the fact that there are no pairs with Lorentz factors above 300 in the model. In order to utilize the reservoir of energy locked up in the form of hot protons to explain the EGRET observations, one must identify an acceleration mechanism that can convert the thermal energy of the protons into electrons with Lorentz factors of $\sim 10^{4}$. Alternatively, one may be able to find a way to directly tap the rotational energy of the black hole itself.

\section{PENROSE PAIR PRODUCTION}

Penrose pair production occurs when the reaction $p+\gamma \rightarrow$ $p+e^{+}+e^{-}$takes place in the ergosphere of a rapidly spinning black hole. In principle, Penrose pair production is an attractive alternative for producing the highest-energy $\gamma$-rays observed from the EGRET AGNs, since the natural energy scale for the process is $m_{p} c^{2}$, where $m_{p}$ is the proton mass (Kafatos 1980; Kafatos \& Leiter 1979). The characteristic timescale for any Penrose process is the light-travel time in the ergosphere, which is $\sim 9$ days for a $10^{10} M_{\odot}$ black hole. This is similar to the observed variability timescale for the EGRET emission from 3C 279. Roughly speaking, in order for a candidate process to be a successful Penrose process (tapping the rotational energy of the hole), the process must produce particles with deflection angles exceeding $90^{\circ}$ and energies comparable to the energy of the projectile particle.

Previous results for photon-proton pair production $(p+$ $\gamma \rightarrow p+e^{+}+e^{-}$) in black-hole ergospheres were obtained by scaling the photon spectrum resulting from the related process of Penrose Compton scattering (Piran \& Shaham 1977). We have recently completed an investigation of Penrose pair production from first principles, utilizing the correct quantummechanical differential cross section for the process. We find that the strong forward peak in the reaction cross section as compared to the Klein-Nishina cross section for Compton scattering effectively prevents large-angle pair production, making it extremely unlikely that Penrose pair production occurs with an astrophysically significant rate. However, the 

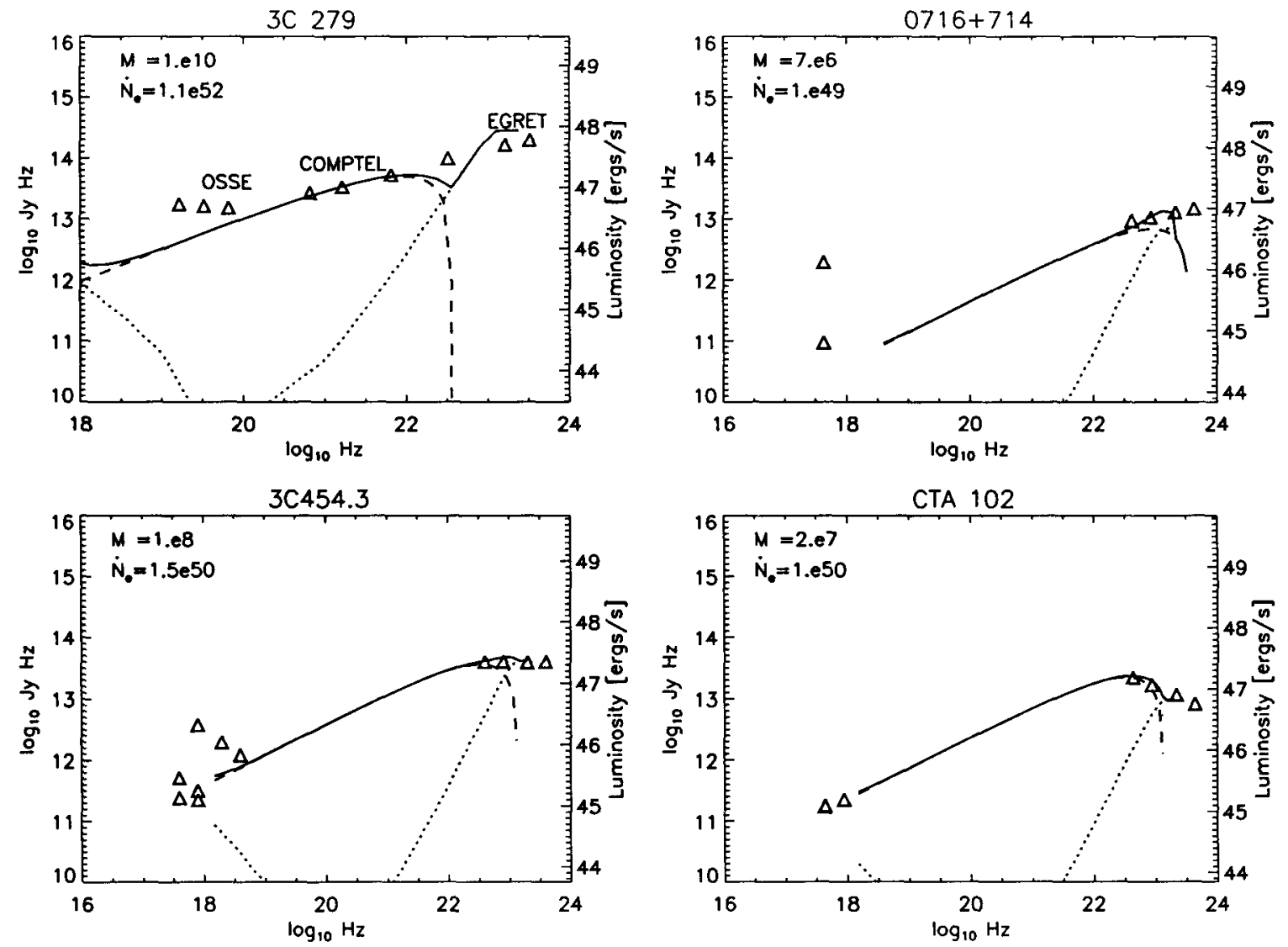

FIG. 1.-Multifrequency observations of four EGRET blazars are shown along with theoretical spectra computed using the simplified particle transport model including pion-decay pair injection, inverse-Compton losses, and escape at the speed of light. Note that stochastic acceleration has not been included. Shown are the spectral components due to the pion-decay $\gamma$-rays (dotted lines) and relativistic Comptonization (dashed lines), as well as the total theoretical spectra (solid lines). The corresponding values of the black hole mass $M$ (solar masses) and the relativistic pair injection rate $\dot{N}_{e}$ (per second) are indicated.

probable elimination of Penrose pair production does not preclude the existence of alternative high-energy hadronic processes that may satisfy the energy and deflection requirements of successful candidate Penrose processes, and we have in fact already identified one possibility, involving the production of resonantly excited nuclei as a result of high-energy proton-proton collisions.

\section{PARTICLE ACCELERATION IN HOT DISKS}

The relativistic pairs created via charged pion decays in the hot inner region of a two-temperature disk are injected with a maximum Lorentz factor $\sim 300$, and therefore in the absence of any additional acceleration mechanism, the inverse-Compton and bremsstrahlung emissions from these pairs appear at energies below $\sim 150 \mathrm{MeV}$. One possibility is that the pion decay pairs are accelerated to much higher Lorentz factors by a collective instability that taps the energy of the hot protons. In this section we show that the duration and the total energy of the 3C 279 EGRET outburst can both be understood if hot protons provide the underlying energy source. We also attempt to constrain the basic parameters of a simple stochastic acceleration model, and discuss the implications for the thermal and dynamical structure of the disk.

The observed $\gamma$-ray luminosity of $3 \mathrm{C} 279\left(\sim 10^{48} \mathrm{ergs} \mathrm{s}^{-1}\right)$ implies a black-hole mass of at least $10^{10} M_{\odot}$ if the emission is isotropic and sub-Eddington. The observed lifetime of $\sim 1$ week implies a total energy $E \sim 10^{54} \mathrm{ergs}$. If one assumes that the emission arises in the (roughly spherical) hot inner region of a two-temperature accretion disk with proton temperature $T_{p} \sim 10^{13} \mathrm{~K}$ and proton number density $n_{p} \sim 10^{8} \mathrm{~cm}^{-3}(\mathrm{EK})$, then it follows from the value of $E$ that the radius of the emission region $r_{\text {hot }} \sim 5 r_{g}$, where $r_{g}=G M / c^{2}$ and $M=10^{10} M_{\odot}$. Hence the outbursts could be powered by the rapid cooling of the hot protons via some nonthermal collective instability that feeds the energy preferentially into the pion decay pairs, accelerating them to Lorentz factors of $\sim 10^{4}$. The accelerated pairs produce $\mathrm{GeV} \gamma$-rays by upscattering soft photons. Since the energy that powers the outburst is provided by the hot protons, the duration of the transient must be less than the accretion timescale so that the protons cool before they cross the horizon. Consequently the two-temperature existence criterion is violated during the $\gamma$-ray flare, and therefore the theory strongly predicts that the flares must be time-dependent. The observed outburst duration of 1 week can be made consistent with the accretion timescale for the hot region if the viscosity parameter $\alpha \lesssim 1$.

We have examined Coulomb collision processes and the associated transport and loss timescales in the hot disk environment, and we have identified particle acceleration as a result of 
collisions between electrons (or positrons) and Alfvén waves in the turbulent disk as a possible mechanism for boosting the Lorentz factors of the pion-decay pairs. The Alfvén waves may be the result of MHD cascades driven on large scales by hydrodynamical eddies, or they may be generated on much smaller scales by transrelativistic thermal protons streaming along the magnetic field lines. Here we hypothesize that one (or both) of these processes results in the production of an intense field of MHD microturbulence, and investigate the implications for the spatial diffusion coefficient and for the thermal and dynamical structure of the disk. Using the usual relationship between the energy-diffusion coefficient $D$ and the spatial diffusion coefficient $\kappa$, we can write (e.g., Becker 1992; Skilling 1975)

$$
\kappa D=\frac{v_{\mathrm{A}}^{2} \gamma^{2}}{9}
$$

for particles with Lorentz factor $\gamma$, where $v_{\mathrm{A}}$ is the Alfvén velocity and the numerical coefficient is approximate. The mean second-order (stochastic) Fermi acceleration rate can be estimated using

$$
\langle\dot{\gamma}\rangle_{\mathrm{F}}=\frac{4 D}{\gamma}
$$

which is exact for momentum-independent spatial diffusion. Equation (2) can be reexpressed in terms of $\kappa$ by substituting for $D$ using equation (1) and setting $v_{\mathrm{A}}=B / \sqrt{4 \pi \rho}$, yielding

$$
\langle\dot{\gamma}\rangle_{\mathrm{F}}=\frac{B^{2} \gamma}{9 \pi \rho k}
$$

where $\rho$ is the mass density.

The combined synchrotron/inverse-Compton loss rate for the relativistic electrons and positrons is related to the timeaveraged magnetic field $B$ and to the isotropic soft-photon (UV/X-ray) energy density $U_{\mathrm{ph}}$ by

$$
\langle\dot{\gamma}\rangle_{\mathrm{loss}}=-\frac{4 \sigma_{\mathrm{T}}}{3 m_{e} c}\left(U_{B}+U_{\mathrm{ph}}\right) \gamma^{2}
$$

where $\sigma_{\mathrm{T}}$ is the Thomson cross section, $m_{e}$ is the electron mass, $c$ is the speed of light, and $U_{B}=B^{2} /(8 \pi)$ is the magnetic energy density. A balance between stochastic acceleration and radiative losses occurs at the critical Lorentz factor

$$
\gamma_{c} \equiv \frac{2 m_{e} c}{3 \sigma_{\mathrm{T}} \rho \kappa_{c}}\left(1+\frac{U_{\mathrm{ph}}}{U_{B}}\right)^{-1}
$$

where $\kappa_{c} \equiv \kappa\left(\gamma_{c}\right)$ is the spatial diffusion coefficient evaluated at $\gamma=\gamma_{c}$. For the turbulent accretion disks of interest here, we expect the magnetic field to be in rough equipartition with the ions, yielding field strengths on the order of $B \sim 10^{3} \mathrm{G}$ if the proton number density $n_{p} \sim 10^{8} \mathrm{~cm}^{-3}$. Since disks with peak temperatures of $\sim 10^{13} \mathrm{~K}$ radiate near-Eddington UV luminosities (EK), we also expect the soft radiation to be in equipartition with the ions, and therefore $U_{\mathrm{ph}} / U_{B} \approx 1$. The value of the spatial diffusion coefficient required in order to achieve $\gamma_{c}=$ $10^{4}$ is then $\kappa_{c} \sim 10^{19} \mathrm{~cm}^{2} \mathrm{~s}^{-1}$, implying a mean free path for the relativistic electrons $l=3 \kappa_{c} / c \sim 10^{9} \mathrm{~cm}$. Taking $B \sim 10^{3}$ $\mathrm{G}$ as an estimate for the magnetic field strength in the hot region, we find that the Larmor radius $r_{\mathrm{L}}=\gamma m_{e} c^{2} /(e B) \sim 10^{4}$ $\mathrm{cm}$ for pairs with Lorentz factor $\gamma=\gamma_{c}=10^{4}$, where $e$ is the electron charge. This is five orders of magnitude smaller than the mean free path, and therefore the value obtained for $\kappa_{c}$ safely exceeds the minimum (Bohm) diffusion coefficient, $\kappa_{\text {Bohm }}=r_{\mathrm{L}} c / 3$ (Krall \& Trivelpiece 1986).

Electrons with Lorentz factor $\gamma$ will upscatter soft photons with incident energy $\epsilon_{0} \ll \gamma m_{e} c^{2}$ to an average energy $\epsilon=(4 /$ 3) $\epsilon_{0} \gamma^{2}$ (Rybicki \& Lightman 1979). The maximum possible energy of the scattered photons is $\epsilon_{\max }=\gamma m_{e} c^{2}$ for fixed $\gamma$, and in order to achieve this, we must set $\epsilon_{0}=(3 / 4) m_{e} c^{2} / \gamma$, or $\epsilon_{0}=$ $38 \mathrm{eV}$ for $\gamma=\gamma_{c}=10^{4}$. This implies a soft photon frequency $\nu_{0}=\epsilon_{0} / h \sim 10^{16} \mathrm{~Hz}$, and a scattered photon energy $\epsilon \sim 5 \mathrm{GeV}$. The mean free time between collisions involving relativistic electrons (or positrons) and soft photons is

$$
t_{\mathrm{ph}}=\left(n_{\mathrm{ph}} \sigma_{\mathrm{T}} c\right)^{-1}
$$

where the soft photon number density $n_{\mathrm{ph}}=U_{\mathrm{ph}} / \epsilon_{0}$, with $U_{\mathrm{ph}}=U_{B}=B^{2} /(8 \pi)$ and $B=10^{3} \mathrm{G}$ from the equipartition arguments. For $\epsilon_{0}=38 \mathrm{eV}$, this yields $t_{\mathrm{ph}} \sim 0.1 \mathrm{~s}$, which is comparable to the characteristic acceleration time,

$$
t_{\mathrm{accel}} \sim \frac{\gamma}{\langle\dot{\gamma}\rangle_{\mathrm{F}}} \sim \frac{9 \pi \rho \kappa_{c}}{B^{2}}
$$

We therefore conclude that most of the pion decay pairs are accelerated to roughly the critical Lorentz factor $\gamma_{c}=10^{4}$ before colliding with a soft photon, and that a complete acceleration/inverse-Compton cooling cycle takes $\sim 0.1 \mathrm{~s}$. The average luminosity per electron (or positron) is therefore $\dot{E}=$ $\gamma_{c} m_{e} c^{2} / t_{\mathrm{ph}} \sim 0.1$ ergs $\mathrm{s}^{-1}$, and it follows that $\sim 10^{49}$ pairs must be experiencing strong turbulence at any given time in order to account for the observed $\gamma$-ray luminosity of $10^{48}$ ergs $\mathrm{s}^{-1}$.

Since the ultrarelativistic electrons and positrons are accelerated at the expense of the hot protons, the number of protons cooling (i.e., driving MHD turbulence) at any given time is also $\sim 10^{49}$. If the hot region has a radius $r_{\text {hot }} \sim 5 r_{g}$ and the proton number density is $n_{p} \sim 10^{8} \mathrm{~cm}^{-3}$, then this represents a fraction $f \sim 10^{-7}$ of the protons, which can also be interpreted as a volume filling factor for the turbulence. The corresponding linear scale is $\sim 10^{-2} r_{g}$ for a $10^{10} M_{\odot}$ black hole. Note that the timescales for electron-electron and electron-proton collisions are each on the order of the accretion time $t_{\mathrm{acc}} \sim 10^{6} \mathrm{~s}$ ( since the two-temperature plasma must be collisionless), and therefore losses due to particle-particle collisions are not likely to play a significant role during the $\gamma$-ray transient. Indeed, the whole point is that the cooling of the hot protons must occur via a collective, collisionless instability in order to produce predominantly high-energy pairs. The requirement of $\gamma$-ray transparency can be satisfied if the $\gamma$-rays are emitted in the funnel of the accretion disk, where they are shielded from the soft radiation produced in the outer regions.

\section{CONCLUSIONS}

In the absence of additional acceleration mechanisms, relativistic electrons and positrons created in the hot region of a 
two-temperature accretion disk produce inverse-Compton emission up to a maximum energy of $\sim 150 \mathrm{MeV}$. This emission may be relevant for the spectrum detected by COMPTEL during the 1991 June flare of 3C 279, but the maximum energy is too low to account for the EGRET observations. This has motivated us to explore alternative means for producing and/ or accelerating ultrarelativistic electrons and positrons up to Lorentz factors of $10^{4}$. The two possibilities considered here are Penrose pair production in the black-hole ergosphere and stochastic acceleration in the hot accretion disk. We find that Penrose pair production is unlikely to occur at an astrophysically significant rate, although the possibility still exists that useful alternative reactions can be found.

The situation with regard to stochastic acceleration in the disk itself is more promising. In particular, we find that the required diffusion coefficient is well in excess of the minimum (Bohm) value, and that the required level of MHD microturbulence may be generated by either large-scale hydrodynamical turbulence or by small-scale streaming instabilities involv- ing the hot ions. The production of $\gamma$-rays via the stochastic acceleration of electrons is certainly energetically plausible, since it directly taps the reservoir of energy locked up in the form of hot protons, which is sufficient to explain the luminosities and lifetimes of the AGN transients detected by EGRET.

We reiterate that the observed high degree of alignment is not inconsistent with a disk origin for the $\gamma$-rays, since the $\gamma-$ $\gamma$ pair production optical depth is expected to increase rapidly as a function of the propagation angle measured relative to rotation axis. In fact, the hot-disk model predicts an anticorrelation for emission above and below $\sim 1 \mathrm{MeV}$ due to $\gamma-\gamma$ pair production, which generally contradicts the predictions of the beam models. This prediction can be tested observationally.

We wish to thank M. Rees for useful criticisms of an earlier draft. This research was supported by a NASA CGRO Phase 2 grant.
Becker, P. A. 1992, ApJ, 397, 88

Begelman, M. C., \& Chiueh, T. 1988, ApJ, 332, 872

Begelman, M. C., Sikora, M., \& Rees, M. J. 1987, ApJ, 313, 689

Eilek, J. A. 1980, ApJ, 236, 664

Eilek, J. A., \& Kafatos, M. 1983, ApJ, 271, 804 (EK)

Hartman, R. C., et al. 1992, ApJ, 385, L1

Hermsen, W., et al. 1993, A\&AS, in press

Kafatos, M. 1980, ApJ, 236, 99

Kafatos, M., ed. 1988, Supermassive Black Holes (Cambridge Univ. Press)

Kafatos, M., \& Leiter, D. 1979, ApJ, 229, 46

Kormendy, L. 1988, in Supermassive Black Holes, ed. M. Kafatos (Cambridge Univ. Press), 98

- 1991, in Testing the AGN Paradigm, ASP Conf. Ser. 254, ed. S. Holt (New York: AIP), 23

Krall, N. A., \& Trivelpiece, A. W. 1986, Principles of Plasma Physics (San Francisco Press)

\section{REFERENCES}

Lynden-Bell, D. 1969, Nature, 223, 690

Maisack, M., Becker, P. A., \& Kafatos, M. 1994, ApJS, in press

Piran, T. \& Shaham, J. 1977, Phys. Rev. D., 16, 1615

Pringle, J. E. 1981, ARA\&A, 19, 137

Rees, M. J., 1984, ARA\&A, 22, 471

Rees, M. J., Begelman, M. C., Blandford, R. D., \& Phinney, E. S. 1982, Nature, 295, 17

Rybicki, G. B., \& Lightman, A. P. 1979, Radiative Processes in Astrophysics (NY: Wiley)

Shakura, N. I., \& Sunyaev, R. A. 1973, A\&A, 24, 337

Shapiro, S. L., Lightman, A. P., \& Eardley, D. M. 1976, ApJ, 204, 187 (SLE)

Skilling, J. A. 1975, MNRAS, 172, 557 